\title{
Proof of concept simple conductive thread stitch sensor to measure the duration of kangaroo care
}

\begin{abstract}
Throughout the world thousands of infants are born each day according to the World Health Organization. All infants can benefit from the use of Kangaroo Care (KC) or bare skin-to-skin contact between caregiver and infant. One of the key principles to getting the most from $\mathrm{KC}$ is that is must be performed for at least one hour according to the World Health Organization. Yet, the duration of $\mathrm{KC}$ is rarely measured. This study explores the use of a simple device, a conductive thread stitch sensor coupled with a pair of magnets and a microcontroller, to measure the duration of $\mathrm{KC}$ automatically. For this study, a single subject used the sensor with a specific $\mathrm{KC}$ garment and performed $\mathrm{KC}$ with a weighted doll. It was determined, that when comparing manual timed values of the duration of $\mathrm{KC}$ to the sensor values, there was $6.43 \pm 5.67 \%$ average percentage difference. This proof of concept shows that this simple device can be used to determine the duration of $\mathrm{KC}$ in an effective manner.
\end{abstract}

Keywords: stitch sensor, conductive thread, kangaroo care, bare skin-to-skin contact, bottom coverstitch

\author{
Volume 7 Issue | - 202 |
}

\author{
Michael JWeber,' Abigail Clarke-Sather,' \\ Kelly Cobb, ${ }^{2}$ Lindsay Naylor ${ }^{3}$ \\ 'Department of Mechanical and Industrial Engineering, \\ University of Minnesota - Duluth, Duluth, Minnesota, United \\ States \\ ${ }^{2}$ Department of Fashion and Apparel Studies, University of \\ Delaware, Newark, United States \\ ${ }^{3}$ Department of Geography \& Spatial Sciences, University of \\ Delaware, Newark, Delaware, United States
}

Correspondence: Abigail Clarke-Sather, Department of Mechanical and Industrial Engineering, University of Minnesota - Duluth, Duluth, Minnesota, United States, Tel 218-726-8424, Email abbie@d.umn.edu

Received: October 09, 2020 | Published: February 03, 2021

\section{Introduction}

All infants can benefit from Kangaroo Care (KC). Kangaroo Care is done by placing the naked newborn on the bare chest of the caregiver, which can be any family member but often is the mother. There are many benefits that $\mathrm{KC}$ can provide to the infant and also to the caregiver. Kangaroo Care can also be done in many different situations but one of the main principles to $\mathrm{KC}$ is that is performed for at least one hour. ${ }^{1}$ Despite this, the duration of $\mathrm{KC}$ is normally not tracked by caregivers, either the infant's family or hospital staff if the infant is being cared for in the neonatal intensive care unit (NICU). In order to address to facilitate tracking of $\mathrm{KC}$, breastfeeding, and human milk expression, the March of Dimes created the My NICU Baby ${ }^{\mathrm{TM}}$ app where the mother or family is expected to track the duration of KC. Mothers and hospital staff are already overburdened, KC duration tracking becomes an additional burden. This Introduction explores what makes $\mathrm{KC}$ so beneficial, challenges of performing $\mathrm{KC}$, the principle elements of conductive thread and the $\mathrm{KC}$ duration stitch sensor, and the current research gap.

The World Health Organization (WHO) established several guidelines established in order for both the infant and the mother to get the most out of KC. First, best practices for KC deem it should be performed for at least one hour each time. ${ }^{2}$ This is due to decreasing the amount of time that the infant is disrupted. ${ }^{3}$ However, previous research by the authors shows that many mothers do not meet the onehour threshold, ${ }^{4}$ therefore reducing the benefits that $\mathrm{KC}$ can provide. Additionally, in order for the infant and mother to get the most from $\mathrm{KC}$, the infant must not be clothed, i.e. wearing no more than a diaper and a hat, and the infant's bare chest must be on the bare chest of the mother. While the caregiver is performing $\mathrm{KC}$ with an infant, the caregiver can walk, sit, or stand. Regardless of what physical position the mother is in, clothing that is loose enough to allow the infant to fit inside but also tight enough to ensure that the infant does not slip out is ideal. ${ }^{2}$
Within these best practices, there are also some general guidelines for when it is appropriate to perform KC with an infant. First, KC can be performed with an infant as small as 1200 grams but is generally not performed with infants greater than 2500grams, as these infants tend to be older and move a lot during KC. ${ }^{2}$ Additionally, $\mathrm{KC}$ is recommended to start immediately after birth. ${ }^{5} \mathrm{Also}, \mathrm{KC}$ is an excellent way to maintain temperature for the infant, especially when compared to incubators. However, in order to ensure that the infant does not suffer from hypothermia, the infant should maintain a skin temperature between $36.2^{\circ} \mathrm{C}$ and $37.2^{\circ} \mathrm{C}$ and the temperature should be checked by the caregiver often. ${ }^{6}$

Several studies have shown that mothers who perform KC with their infants, provide short term and long term health benefits to their children. Of the short term benefits, KC can stabilize the respiratory rate of the infant and decrease the number of apnea episodes. ${ }^{7,8}$ Kangaroo Care has similar effects on the infant's heart rate, where $\mathrm{KC}$ increases the stability of the infant's heart rate and decreases the number of bradycardia events. ${ }^{7,8}$ Finally, $\mathrm{KC}$ has been shown to be an effective pain treatment for infants and in one study, the researchers found that $\mathrm{KC}$ was just as effective as $24 \%$ Sucrose.

The benefits of $\mathrm{KC}$ are not just limited to the short term. Kangaroo Care can help improve mothers' abilities to breastfeed. For example, mothers who are not able to breastfeed their children in the NICU, tend to struggle to meet breastfeeding goals once out of the NICU. ${ }^{10,11}$ However, mothers who perform $\mathrm{KC}$ with their children while in the NICU on average breastfeed longer than those who do not perform KC. ${ }^{10,11}$ Increasing the duration an infant is breastfed can reduce early diseases and infections, such as lower respiratory tract infections, and can also reduce later health problems such as diabetes, obesity, and hypertension. ${ }^{12}$ In a recent study, the health of participants was reported for participants that had received KC 20 years earlier as infants. The researchers found that overall mortality over the course of 20 years was lower in the $\mathrm{KC}$ group versus the control group. ${ }^{13}$ 
Kangaroo Care can also have benefits for the mother as well as the child. In two current studies, mothers who perform $\mathrm{KC}$ benefit from a reduction in stress and $\mathrm{KC}$ is an effective method to treat maternal depression and anxiety. ${ }^{14,15}$ Kangaroo Care also encourages mothers to breastfeed, which results in its own health benefits, such as reduced chance of obesity. ${ }^{10,12}$ Since mothers who perform KC are more likely to breastfeed, the health benefits associated with breastfeeding have directly benefited from $\mathrm{KC} .{ }^{8}$

There are however several hurdles that mothers and caregivers face when trying to perform $\mathrm{KC}$, such as nurse attitudes in the NICU, the infant's health, clothing, and lack of $\mathrm{KC}$ duration tracking. One challenge mothers face with regards to $\mathrm{KC}$ is that mothers are not always given enough resources regarding $\mathrm{KC}$ to either correctly perform $\mathrm{KC}$ or to decide whether it is right for them. In a recent study, researchers found that 4 of the top 5 challenges facing mother regarding $\mathrm{KC}$ were resource related. ${ }^{16}$ Additionally, nurses and hospital staff may make KC more difficult instead of easier for the mother. Even though the benefits of $\mathrm{KC}$ are well documented, many nurses and other hospital staff either find $\mathrm{KC}$ unnecessary or are uncomfortable having the mother perform KC.,11,17 One study in 2013 in the US, found that only $18 \%$ of nurses encourage $\mathrm{KC}$ in their NICUs. ${ }^{18}$ Another study done in the US, found that only $8 \%$ of the participants that responded to a national survey reported that $\mathrm{KC}$ was performed in their NICUs. ${ }^{19}$ Coupled with these low percentages is the fact that some nurses that do allow $\mathrm{KC}$ dictate its usage based on their own judgement that may or may not agree with guidelines. ${ }^{3,20}$ Some nurses may disregard guidelines and use their own judgement assessing whether the infant's health condition will allow KC.

The infant's health condition is another barrier to KC. Whether the infant is receiving breathing support, such as ventilation from a jet oscillator, or thermal regulation or light therapy support from an incubator, $\mathrm{KC}$ can still be performed under close supervision and in shorter increments. ${ }^{4}$ However, nurse or other hospital staff might not feel comfortable taking infants out of the controlled environment of the incubator and placing infants skin-to-skin next to their mothers which is an environment with many variables that nurses cannot control. Finally, the clothes that the mother wears can limit how effective $\mathrm{KC}$ can be performed. Clothing impacts how mothers may feel exposed or uncomfortable during KC. ${ }^{11,22}$

Currently mothers can use apps, such as the March of Dimes My NICU Baby ${ }^{\mathrm{TM}}$ app to manually record and track how long they perform $\mathrm{KC}$. However, mothers having to track $\mathrm{KC}$ on their own add additional burdens on them while their children are in the NICU. Additionally, nurses are overburdened with charting and other responsibilities and therefore are not always able to chart KC. Currently there are a few automated systems that measure the duration of $\mathrm{KC}$ on the market. ${ }^{23-25}$ With one such system, the device measured $52 \%$ of the total duration of $\mathrm{KC}$ with a $64 \%$ average percentage difference between the sensor measured durations and manual measured durations over the course of several hours. ${ }^{23}$ This large difference in the time spent in $\mathrm{KC}$ and the time tracked by the sensor makes it difficult to determine whether the infant is gaining the full benefits from KC. Additionally, existing devices are bulky in size and made of a hard plastic, which is uncomfortable for the infant wearer ${ }^{23,24}$ There are other device prototypes that measure other health outcomes, such as heart rate and temperature, of the infant that use conductive elements, ${ }^{25}$ however they do not measure the duration of $\mathrm{KC}$ being performed.

In order to automatically measure how long $\mathrm{KC}$ is performed, a sensor was made of a flexible material that would not deform from body heat. Additionally, the sensor was made to integrate with the
SnuggleTime garment (Figure 1) designed to address privacy and other concerns about clothing from mothers performing $\mathrm{KC}$ in NICUs. The Snuggletime garment was created via a code sign process including interviews with mothers of NICU infants and a focus group of mothers who gave feedback and tried on a garment design during fit testing. ${ }^{22}$ Integration with the stretchy fabric of the undergarment or base layer bandeau piece of the Snuggletime garment meant that the sensor would still need to work with large changes in geometry as the bandeau stretched open to accommodate the infant for $\mathrm{KC}$.

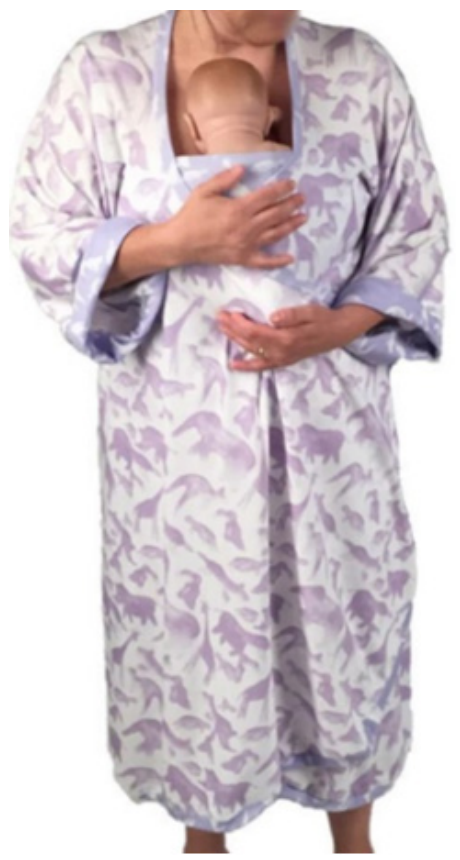

Figure I Image of the SnuggleTime Garment. ${ }^{22}$

Due to this requirement, conductive thread, sewn into a stitch sensor, was used as a flexible sensor to ensure that there was consistent measurement happening for a wide assortment of dimensions, as seen in Figure 2. The use of stitch sensors has already been explored for measuring knee joint angles and motion tracking. ${ }^{26-28}$ Coverstitch was chosen for the stitch sensor because of its greater flexibility than other stitches, which reduced the chance of the conductive thread breaking. Additionally, researchers have demonstrated that when conductive thread is sewn in a bottom cover thread stitch, it has been shown to not break at or past $30 \%$ elongation..$^{27}$ The reason conductive thread in stitch sensors can measure different values is because as the stitch is stretched, the thread becomes more in contact with itself, effectively changing the resistance. ${ }^{29}$ For this study, this stitch sensor is treated as part of a simple ON/OFF switch using a digital pin on an Adafruit Feather microcontroller. In this case, when the switch is on, the magnet is closed, and $\mathrm{KC}$ is not being performed. When the mother holds her infant the fabric is stretched, the switch is off, the magnet is open and $\mathrm{KC}$ is being performed. The changes in resistance due to the stretching of the conductive thread create no effect on the end results.

In order to use a conductive thread stitch sensor, coupled with a pair of magnets, as an ON/OFF switch, several characteristics need to be determined. First, the amount of force necessary to break the pair of magnets needs to be determined. If this force is too great, then the force provided by the displacement of the stitch sensor in the garment when someone is wearing it will not be enough to separate the magnets and the sensor will not record any duration. Conversely, if the force to break the magnets is too weak, then this could cause false positive readings when no infant is being held in KC. Note that in Figure 2, 
the conductive thread connected to the magnets is not parallel with the magnets. Due to this the force to break the magnets needs to be broken into components, as shown in a free body diagram in Figure 3. Second, with a known breaking strength, the displacement of the stitch sensor necessary to generate this force can be calculated. To start to solve for this displacement, a method to relate force to stress is needed as shown in Equation 1.

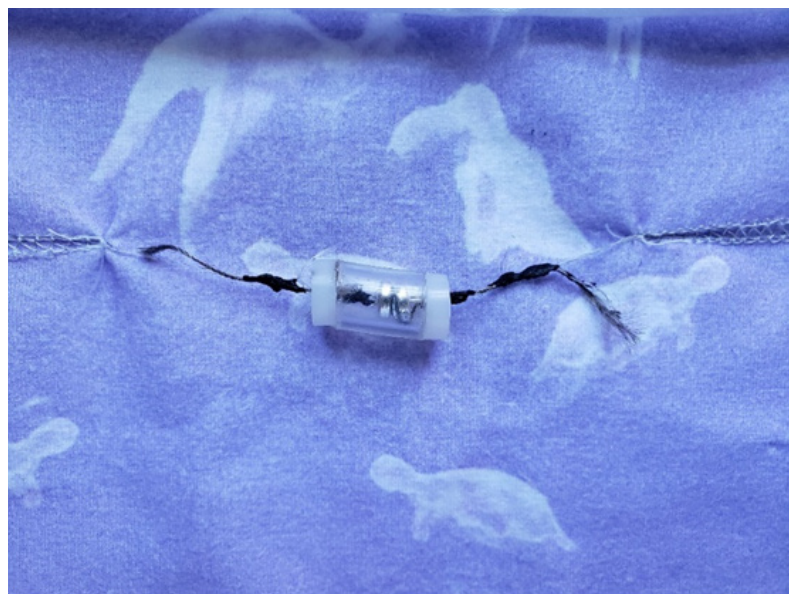

Figure 2 Enclosed magnets attached to the stitch sensor (authors' image).

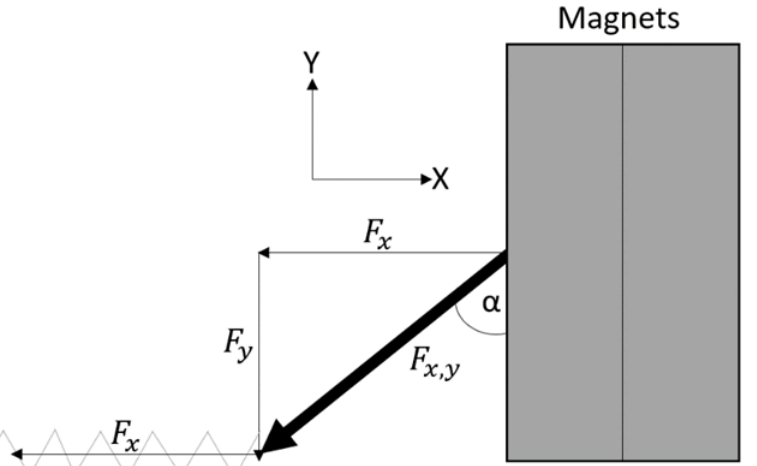

Stitch Sensor

Figure $3 \mathrm{~A}$ free body diagram of the magnets and the stitch sensor (authors' image).

$$
\sigma_{x}=\frac{F_{x}}{A_{x}}
$$

Where $\mathrm{F}_{\mathrm{x}}$ is the force to break the magnets, $\mathrm{A}$ is the cross-sectional area of a stitch sensor, and $\sigma_{x}$ is the stress applied to the stitch sensor from this force. Additionally, Equation 2, Hooke's law, can be used to relate the stress a material exhibits to the strain on the system found via experimental evidence for a specific material, in this case the combination of the stitch sensor on the base fabric.

$$
\sigma_{x}=E \varepsilon_{x}
$$

Where $\mathrm{E}$ is the elastic modulus of the stitch sensor sewn into the fabric and $\varepsilon$ is the strain on the stitch sensor-fabric system. Strain of the system is also defined by Equation 3 .

$$
\varepsilon_{x}=\delta / L
$$

Where $\delta$ is the displacement the stitch sensor exhibits, and $\mathrm{L}$ is the total length of the stitch sensor at rest. Finally, when combining Equations 1-3 for $\delta$, Equation 4 is determined, which defines the displacement of the stitch sensor.

$$
\delta=\frac{F_{x} L}{A_{x} E}
$$

With Equation 4, the displacement that the base fabric, and therefore the stitch sensor, needs to exhibit to separate the magnets can be determined. By using this equation, the sensor can be designed to be sensitive enough to measure infants with low birth weight and size but also not generate false readings.

Research gap: The aim of this study was to construct a sensor (Figure 4) that could automatically record the amount and duration of $\mathrm{KC}$ being performed between mother and infant. This sensor reduces the burden of recording the duration of $\mathrm{KC}$ manually by mothers, families, or nurses. With this type of automatic sensor (Figure 5), it will also be possible to compare how effective different wearables, hospital guidelines, or educational interventions are at facilitating $\mathrm{KC}$.

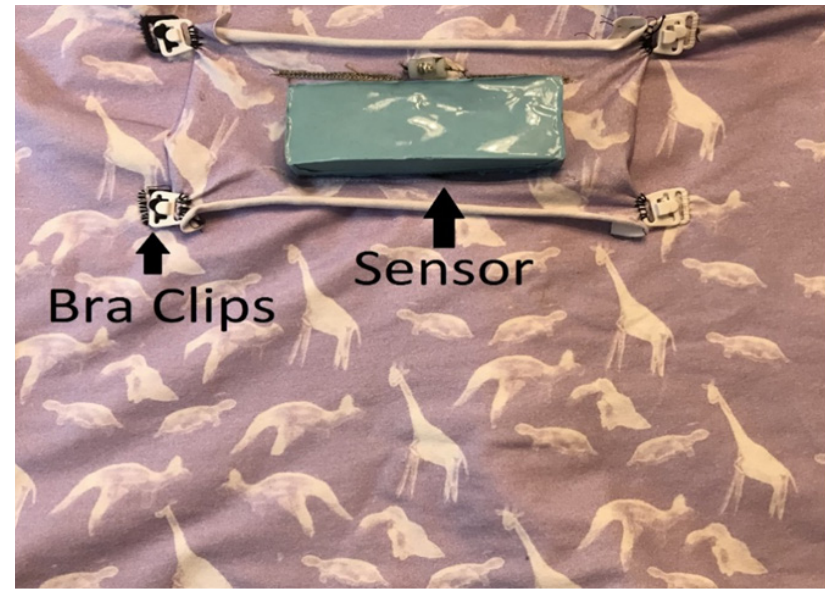

Figure 4 Image of the garment and the sensor attached (authors' image).

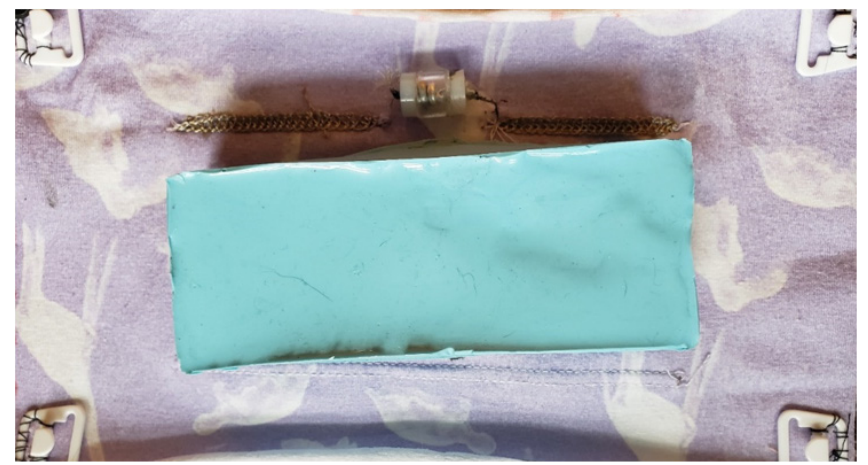

Figure $\mathbf{5}$ Image of the fully assembled KC sensor (authors' image).

\section{Materials and methods}

Several challenges were overcome to create this $\mathrm{KC}$ duration sensor. Firstly, the sensor needed to be sensitive enough that when a small preterm infant is placed on a mother's chest, the fabric will still displace enough for the magnets to pull apart and the sensor to still work. Secondly, the sensor needed to be as small and safe as possible to avoid the possibility of transferring electric current to infants or mothers involved. Finally, the sensor needed to be body-conforming since no mother-baby pair or dyad is shaped exactly the same. A discussion of how the sensor was constructed and how trials were conducted follows.

There were several materials that were used to construct this sensor. For measuring the duration of $\mathrm{KC}$ that was performed the following materials were used; 2 ply silver coated nylon thread of $50 \Omega / \mathrm{m}$ was sewn using a Brother $2340 \mathrm{cv}$ cover stitch machine into a 4 -way stretch $93 \%$ cotton $7 \%$ spandex jersey fabric. The fabric was 
cut into a $14 \mathrm{~cm}$ by $28 \mathrm{~cm}$ rectangle, with the $\mathrm{KC}$ sensor stitches being placed $4 \mathrm{~cm}$ from the top edge and centered in the horizontal direction across the torso. The conductive thread was sewn into two $10 \mathrm{~cm}$ sections with a $4 \mathrm{~cm}$ gap between the two. In this $4 \mathrm{~cm}$ gap, two silver coated magnetic jewelry clasps were placed in $1 \mathrm{~cm}$ clear tubing and sealed with two plastic washers using a plastic adhesive. Once sealed, each magnetic clasp was attached to one of the conductive threads using a clinch knot, as seen in Figure 6, with five to seven wraps used for the conductive thread in the clinch knot. Once connected, these connections were coated with a liquid rubber to ensure that there would be no slipping of the knot. Figure 2 shows the magnets attached to the conductive thread stitches. Once the sensor was finished, four nursing bra clips were attached to each of the corners of the fabric.

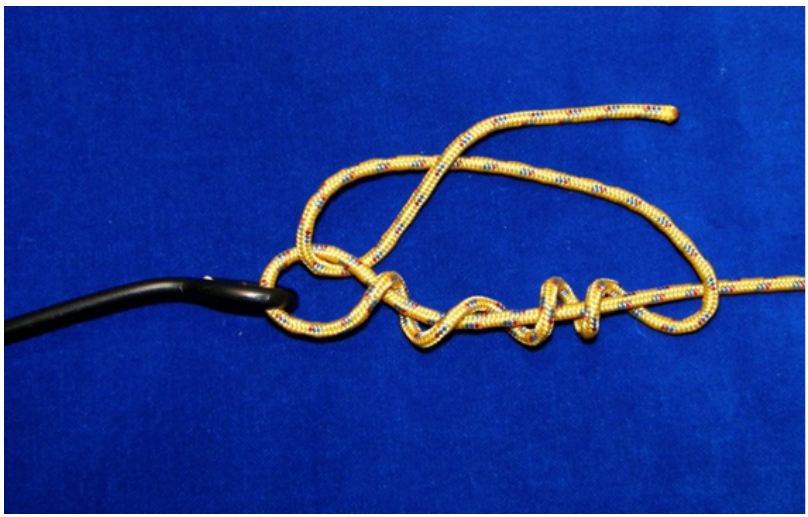

Figure 6 An example of a clinch knot. ${ }^{32}$

The corresponding four clips to connect to were sewn onto the SnuggleTime garment, ${ }^{22}$ as seen in Figure 4, which shows the garment with the sensor attached. The SnuggleTime garment was worn during trials when preforming KC. Once the base of the sensor was constructed, the free ends from the each of the conductive thread stitches were connected to an Adafruit Feather M0 with an Adalogger shield. This sensor was built as an ON/OFF switch for a timer Thus, one of the free ends was connected to a digital terminal and the other end was connected to the ground terminal. Kangaroo Care duration timing was started when the circuit was off and the magnets disconnected and stopped when the magnets and circuit reconnected. For the connections to the Feather M0, the pins were not soldered into the board. Instead the thread was fed through each of the corresponding terminals and a clinch knot was used to connect the thread to the board. Once connected to the board, a small amount of liquid rubber was placed on the connection point to ensure that there was a solid connection. Finally, a blue silicon case was constructed using a mould to protect the Adafruit Feather board. The Feather M0 was then placed into this casing and attached to a $550 \mathrm{mAh}$ battery. In Figure 5 one can see how the sensor looked when fully assembled. Because the Feather M0 has an onboard WIFI module, the results from any test were transmitted to a private ThingSpeak ${ }^{\mathrm{TM}}$ channel. A Huawei Airbox Mobile hotspot was used to create the connection between the $\mathrm{KC}$ sensor and the internet for uploading to the ThingSpeak ${ }^{\mathrm{TM}}$ channel, as seen in Figure 7.

Before the sensor could be tested with a participant, several initial tests needed to be conducted. First, the breaking strength of the magnets needed to be determined. A pair of silver coated magnets, the same ones that were used in the sensor construction, was attached to conductive thread using a clinch knot. Then each of the conductive threads were placed into the grips of a tensile testing machine that is designed for testing small loads, called the MinStrain. ${ }^{30}$ Once placed in the MinStrain, a displacement of $3 \mathrm{~cm} / \mathrm{min}$ was applied to the sample until the magnets separated. Once the magnets separated, the force at that point was recorded. This was repeated five times to find the average breaking force needed. Additionally, the elastic modulus of the stitch sensor needed to be determined. This was determined by placing a $4 \mathrm{~cm}$ by $10 \mathrm{~cm}$ piece of base fabric with a $4 \mathrm{~cm}$ stitch sensor sewn down the middle, into the MinStrain and stretching it at rate of $60 \mathrm{~mm} / \mathrm{min}$. The sample was stretched to about $6 \mathrm{~cm}$. This was done a total of five times and averaged. The resulting stresses and strains were plotted and the secant modulus was determined and converted to find the elastic modulus. Once the elastic modulus and the breaking force were determined, the displacement could be solved using Equation 4. From the displacement, the location of the bra clips on the KC garment was adjusted such that a displacement from a 40 -week gestational age infant was enough to break the magnets.

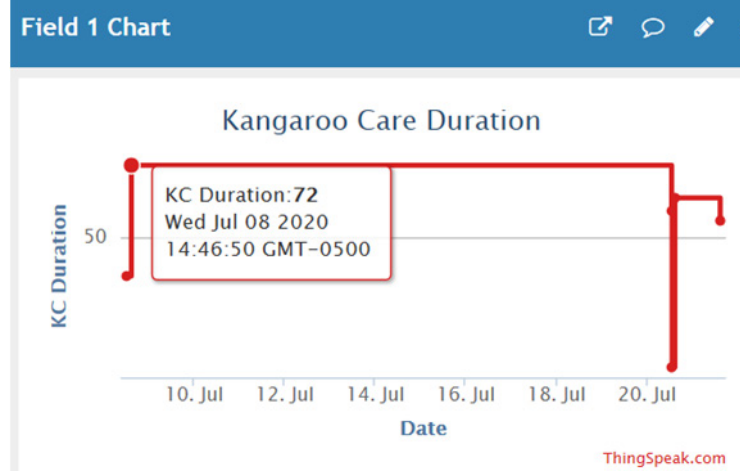

Figure 7 Kangaroo Care duration from sensor sent to ThingSpeak ${ }^{\mathrm{TM}}$

The second preliminary test tested the effectiveness of the sensor under displacement. The stitch sensor was placed in the same tensile testing machine as the previous test and was stretched until the magnets separated, at which point manual timing was started to ensure that the sensor was measuring the correct time. Once separated, the sensor was observed for between 1-2 hours for a total of five trials.

In order to test the effectiveness and accuracy of this sensor, the sensor was tested with a single subject performing $\mathrm{KC}$ with a weighted doll (Figure 8). A single subject research approach was taken in order to validate the proof of concept sensor, similar to. ${ }^{4} \mathrm{~A}$ single subject approach was selected for this study, as it is commonly done with developmental medicine. Additionally, single subject research focuses on repeated trials of single or small sample of subjects and how actions change with repeated usage. ${ }^{31}$

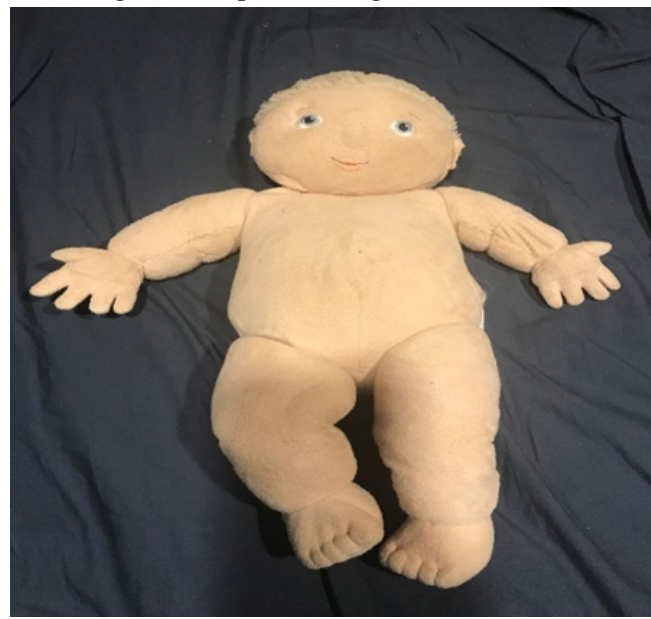

Figure 8 Image of the weighted doll used for the participant trials (authors' image). 
For this study, the participant was part of the research team and thus human subjects research approval did not apply although the research team is working under a UMN IRB approved human subjects research protocol \#STUDY00008380 entitled "Measuring Kangaroo Care Duration with Healthy Adult Women". For this study, the participant was a middle-aged American mother, identifying ethnically as white, who had a BMI of 27.3.

To start, the participant was prompted to don a SnuggleTime garment and place the sensor on the garment with the bra clips. Then, the participant was given a weighted doll, as seen in Figure 8, that represented the approximate size of a child of 40 weeks gestational age, with a length of $46 \mathrm{~cm}$ and head circumference of $36 \mathrm{~cm}$, with a total weight of about $2 \mathrm{~kg}$. Next the participant placed the weighted doll inside the SnuggleTime garment and was instructed to perform $\mathrm{KC}$ will performing normal daily activities, such as sitting, standing, or walking. Once the infant was placed in KC, the participant manually tracked the duration of $\mathrm{KC}$ being performed with a clock and using the March of Dimes My NICU Baby ${ }^{\mathrm{TM}}$ app. After KC was finished in about one hour, the participant would remove the weighted doll from $\mathrm{KC}$, press stop in the My NICU Baby ${ }^{\mathrm{TM}}$ app, and record the final duration from the clock. This was repeated a total of five times.

Once completed, the data was compiled and analysed using Microsoft Excel. Firstly, the average displacement needed to break the magnetic bond was calculated. Also, the average difference between the manually recorded time and the sensor measured time was calculated for the preliminary tests. Also, the average difference between the manual measured durations and sensor measured trials for the participant study were calculated.

\section{Results and discussion}

\section{Preliminary trials}

From the first part of the preliminary trials, the average breaking strength of the magnets for this sensor was found to be $2.21 \pm 0.33$ $\mathrm{N}$. Using this value, in combination with Equation 4 and with the length of the sensor measured as $14 \mathrm{~cm}$, the elastic modulus of the fabric calculated at $40.21 \mathrm{kPa}$, and the cross sectional area of a stitch sensor measured at $0.0014 \mathrm{~m}^{2}$, the displacement necessary to break the magnets was determined to be $5.9 \mathrm{~mm}$. Note that the cross sectional area of the stitch sensor was the thickness of the stitch and base fabric, measured at $1.49 \mathrm{~mm}$, multiplied with the width of the stitch, measured at $1 \mathrm{~cm}$. Based on this displacement value, the placement of the bra clips on the garment were initially adjusted to about the length of the sensor, which was $28 \mathrm{~cm}$.

From the second part of the preliminary trials the duration from the sensor and the manual recorded durations were determined. In Figure 9, the manual recorded durations and the sensor durations for five trials are shown. Note that the black line in Figure 9 indicates the expected relation of one to one match between the two durations. These trials were done over the course of one to two hours. The average percentage difference between sensor duration and manual tracked duration was $0.47 \pm 0.28 \%$ or $0.45 \pm 0.27$ mins.

This minimal difference between manual and sensor recorded tracked time shows that this $\mathrm{KC}$ sensor is an effective method to measure the duration of $\mathrm{KC}$ when compared to manual recorded durations. It should be noted that during one of the trials, the $\mathrm{KC}$ sensor slipped through the grips of the MinStrain and resulted in the time from the KC sensor being less than the manual time. Due to this mishap, that trial was deemed a outlier and excluded from the results shown in Figure 8. Additionally, it was determined that the power draw from the Feather M0 to run the code from the sensor was $41.66 \mathrm{mAh}$, giving the life of the sensor a theoretical total of 13.2 hours with the current setup and achieving full charge after about 2 hours.

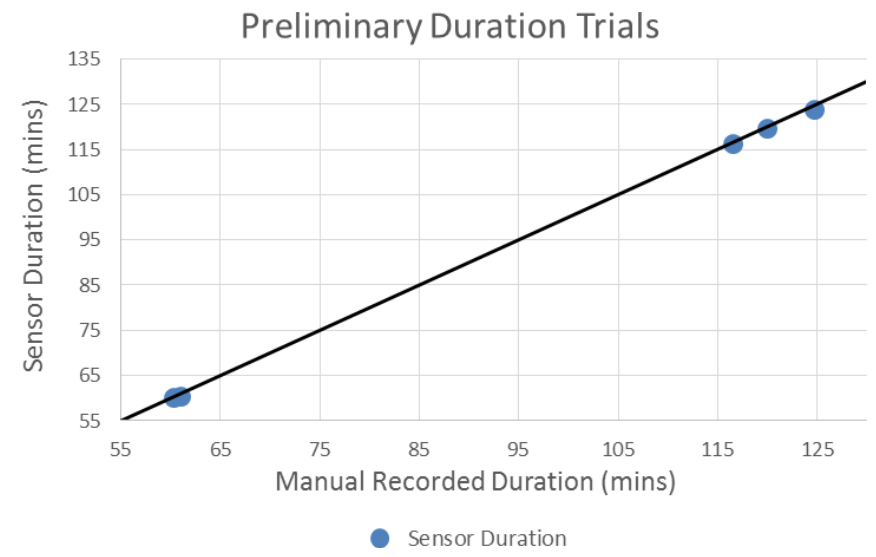

Figure $9 \mathrm{KC}$ sensor initial preliminary duration results.

\section{Participant trials}

From the participant trials, Figure 10 shows the KC durations measured using the sensor, the My NICU Baby ${ }^{\mathrm{TM}}$ app, and a manual recorded duration. Note that the black line indicates a one to one relation between the manual and other recorded durations. For these trials, the clock time was treated as the actual time and thus the average amount of $\mathrm{KC}$ duration was $61.2 \pm 16.3$ minutes. The average difference between the sensor and the My NICU Baby ${ }^{\mathrm{TM}}$ app was $4.98 \pm 5.41 \%$ or $3.04 \pm 3.31$ minutes. The average percentage difference between the sensor and the manual recorded durations was $6.43 \pm 5.67 \%$ or $3.93 \pm 3.47$ minutes. Finally, the difference between the My NICU Baby ${ }^{\mathrm{TM}}$ app and the manual recorded durations was $1.83 \pm 1.92 \%$ or $1.12 \pm 1.17$ minutes.

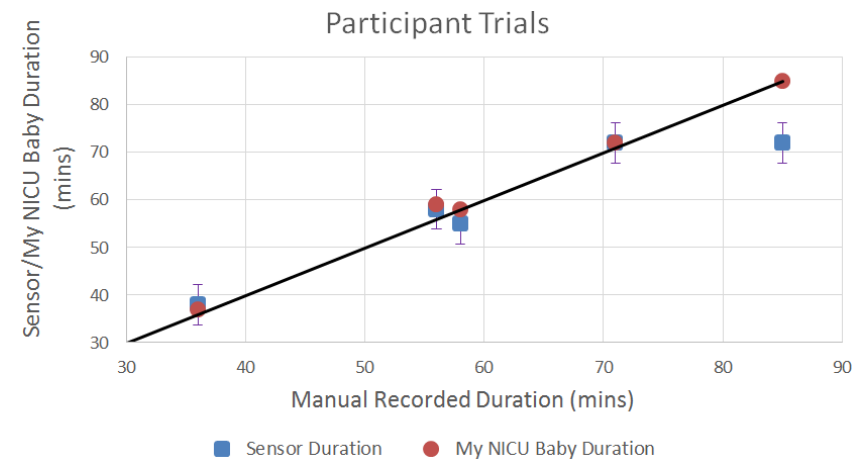

Figure 10 Single participant $K C$ sensor duration results.

These results are a great improvement to the values found in, ${ }^{23}$ which reported an average percentage difference of $64.1 \%$ between sensor and manual recorded time. The percentage difference could have been even smaller with the exclusion of trial 4 , during which the magnet closed during the trial, stopping the timer one time. The magnets closed due to the participant adjusting/rearranging the garment position on the body with the weighted doll, something that is likely to happen during normal use of the garment and integrated stitch sensor. Despite the magnet closing, the sensor timer still measured a duration that was within 15 minutes of the other timed durations as the magnet reopened and started recording again. This temporary closure of the magnet can be addressed via post data processing or rewriting of the code to accommodate short (minute long or less) closing and reopening of the magnets. 


\section{Sensor problems and errors}

There are several sources of error and problems that arose from this study. First, the displacement calculated to pull apart the magnets was determined to be $5.9 \mathrm{~mm}$. However, there was a large amount of slack in between the stitch sensor and the magnets. This resulted in the amount of displacement needed to open the magnets to be $6 \mathrm{~cm}$ on each side of the garment, which was achieved by adjusting the placement of the bra clips sewn on the SnuggleTime garment. However, this pre-strain on the stitch sensor's fabric base made the sensor rather uncomfortable to wear as it applied pressure on the participant's body. Also, at one point during the study the SIM card used for the mobile hotspot which was providing the WIFI for uploading KC duration to ThingSpeak ${ }^{\mathrm{TM}}$ ran out of memory. This caused several trials to not be reported to ThingSpeak ${ }^{\mathrm{TM}}$, which therefore were not included in the reported results. Additionally, based on the code and the hardware of the sensor, the $550 \mathrm{mAh}$ battery that was used for the circuit should have lasted 13.2 hours. However, during several trials, the battery died during usage, resulting in no trial being recorded. In total 6 trials were not recorded due to these two problems. Another issue with the current setup was that the mobile hotspot had to be turned on and off depending on when it was being used in order to save battery life, which became burdensome for the user. Finally, during trial 4 of the participant trials, the participant noted that the magnet had closed during use when readjusting the garment and weighted baby. This caused two entries to be reported to ThingSpeak ${ }^{\mathrm{TM}}$ and resulted in a larger percentage difference between the sensor and the manual recorded durations and My NICU Baby ${ }^{\mathrm{TM}}$ app.

\section{Conclusion}

This study demonstrated a proof of concept for a stitch sensor to automatically measure the duration of $\mathrm{KC}$. It was determined that the percentage difference between a manual recorded duration and this sensor with a participant was $6.43 \pm 5.67 \%$. Additionally, it was determined that the percentage difference between this sensor and the time recorded from the My NICU Baby ${ }^{\mathrm{TM}}$ app was $4.98 \pm 5.41 \%$. These results represent a significant improvement over other methods. This proof of concept has promise to more accurately automatically measure $\mathrm{KC}$ duration than currently available methods. With the use of this sensor, $\mathrm{KC}$ duration can be tracked without burdening caregivers such as mothers and nurses and can even be used by caregivers outside of the hospital. Automatic KC duration tracking facilitated by this sensor enabled garment will allow for the further study of the benefits of $\mathrm{KC}$ and exploration of how duration of $\mathrm{KC}$ impacts different health outcomes.

\section{Future work}

Even though the results from this study show successful proof of concept for a stitch sensor to measure $\mathrm{KC}$ duration, there are several additional aspects that could be looked at in the future. First, the end goal of this study is to have a sensor that could be used by any mother-infant dyad. In order to accomplish this study needs to be done with multiple mother-infant dyads to see how effective this sensor is in a real-world environment especially with a moving infant. Secondly, as stated in the Sensor Problems and Errors section, the battery capacity and SIM card life caused several trials to not be recorded to ThingSpeak ${ }^{\mathrm{TM}}$. In order to avoid this in the future, a more energy efficient sensor needs to be constructed. A custom-built microcontroller that can have better power regulation methods with regards to WIFI protocols could potentially save energy. Additionally, a larger SIM card needs to be placed in the mobile hotspot to ensure hundreds of trials can be run before it needs replacement. Additionally, the integration of other stitch sensors could be used to measure other vital signs recorded in the NICU, such as respiratory rate, heart rate, and temperature. Finally, as previously discussed the sensor was generally uncomfortable when being worn most likely due to the pre-strain needed for the sensor to function. The bra clips used for attachment of the sensor likely did not provide an even distribution of the force from the garment to the sensor exacerbating this problem. In future iterations, the sensor will be

1) Designed to respond without pre-straining the base fabric by making the connecting thread between the magnets and the stitch sensor tighter.

2) Connected to the garment via alternative means such as a zipper or hook and loop fasteners on both sides to ensure more even distribution of the force.

\section{Acknowledgments}

Thank you to the many mothers and hospital staff who care for infants in the NICU whose insights have informed this research.

\section{Data availability}

The data used to support the findings of this study are accessible by contacting corresponding author Abigail Clarke-Sather.

\section{Funding}

There was no external funding for this project.

\section{Conflicts of interest}

The authors declare that there is no conflict of interest regarding the publication of this paper.

\section{References}

1. Ludington-Hoe SM. A clinical guideline for implementation of kangaroo care with premature infants of 30 or more weeks' Postmenstrual Age. Advances in neonatal care. 2008;8:S3-S23.

2. World Health Organization. Department of Reproductive Health and Research. Kangaroo mother care: a practical guide. 2003.

3. Naylor L, Clarke-Sather AR. Mothers' Intention to Breastfeed in the NICU and Factors Effecting Duration of Milk Expression. International Journal of Caring Sciences. 2020.

4. Clarke-Sather AR, Cobb K, Maloney C, et al. Contextual design theory applied to wearables that facilitate kangaroo care by interviewing mothers of hospitalized infants. MN, USA: 2018 Design of Medical Devices Conference, Minneapolis; 2018.

5. Crenshaw JT. Healthy birth practice \#6: keep mother and baby together- It's best for mother, baby, and breastfeeding. J Perinat Educ. 2014;23(4):211-217.

6. Knobel RB. Thermal stability of the premature infant in neonatal intensive care. Newborn and Infant Nursing Reviews. 2014;14(2):72-76.

7. Ludington-Hoe S, Anderson GC, Swinth J, et al. Randomized controlled trial of kangaroo care: cardiorespiratory and thermal effects on healthy preterm infants. Neonatal Network. 2004;23(3):39-48.

8. Ludington-Hoe SM. Evidence-based review of physiologic effects of kangaroo care. CWHR. 2011;7(3):243-253.

9. Campbell-Yeo M, Celeste Johnston C, Benoit B, et al. Sustained efficacy of kangaroo care for repeated painful procedures over neonatal intensive care unit hospitalization: a single-blind randomized controlled trial. PAIN. 2019;160(11):2580. 
10. Briere CE, McGrath J, Cong X, et al. An integrative review of factors that influence breastfeeding duration for premature infants after NICU hospitalization. Journal of Obstetric, Gynecologic, \& Neonatal Nursing. 2014;43(3): 272-281.

11. Naylor L, Clarke-Sather AR. Factors impacting breastfeeding and milk expression in the neonatal intensive care unit. International Journal of Caring Sciences. 2020.

12. Binns C, Lee M, Low WY. The long-term public health benefits of breastfeeding. Asia Pac J Public Health. 2016;28(1):7-14

13. Charpak N, Rejean T, Juan GR, et al. Twenty-year Follow-up of Kangaroo Mother Care Versus Traditional Care. Pediatrics. 2017;139(1):e20162063.

14. Chiu SH, Anderson GC. Effect of early skin-to-skin contact on motherpreterm infant interaction through 18 months: randomized controlled trial. Int J Nurs Stud. 2009;46(9):1168.

15. Cho ES, Shin-Jeong K, Myung SK, et al. The effects of kangaroo care in the neonatal intensive care unit on the physiological functions of preterm infants, maternal-infant attachment, and maternal stress. Journal of Pediatric Nursing. 2016;31(4):430-438.

16. Seidman G, Shalini U, Emma K, et al. Barriers and enablers of kangaroo mother care practice: a systematic review. PLOS ONE. 2015;10(5):e0125643.

17. Nimbalkar S, Sadhwani N. Implementation of kangaroo mother care challenges and solutions. Indian Pediatr. 2019;56(9):725-729.

18. Hendricks-Muñoz KD, Li Y, Kim YS, et al. Maternal and neonatal nurse perceived value of kangaroo mother care and maternal care partnership in the neonatal intensive care unit. American Journal of Perinatology. 2013;30(10):875-880.

19. Cooper LG, Gooding JS, Gallagher J, et al. Impact of a family-centered care initiative on NICU care, staff and families. J Perinatol. 2007;27:S32S37.

20. Engler AJ, Susan MLH, Regina MC, et al. Kangaroo care: national survey of practice, knowledge, barriers, and perceptions. MCN: The American Journal of Maternal/Child Nursing. 2002;27(3):146-153.
21. Karlsson H. Skin to skin care:heat balance. Archives of Disease in Childhood - Fetal and Neonatal Edition. 1996;75(2):F130-F132.

22. Cobb KA, Clarke-Sather AR. Empathetic iteration of a snuggletime garment system for kangaroo care of mothers and babies in the neonatal intensive care unit. Journal of Textile and Apparel, Technology and Management. 2020.

23. Rao S, Thankachan P, Amrutur B, et al. Continuous, real-time monitoring of neonatal position and temperature during Kangaroo Mother Care using a wearable sensor: a techno-feasibility pilot study. Pilot Feasibility Stud. 2018;4(1):99.

24. Joglekar A, Alok R, Vasanth R, et al. A wearable sensor for monitoring kangaroo mother care treatment for premature neonates. IEEE SENSORS. 2018:1-4.

25. Chen W, Bambang Oetomo S, Feijs L, et al. Design of an integrated sensor platform for vital sign monitoring of newborn infants at neonatal intensive care units. Journal of Healthcare Engineering. 2010;1(4):535-553.

26. Chen H, Xue M, Mei Z, et al. A review of wearable sensor systems for monitoring body movements of neonates. Sensors. 2016;16(12):2134.

27. Gioberto G, Compton C, Dunne L. Machine-stitched E-textile stretch sensors," sens. Transducers J. 2016;202:25-37.

28. Dupler E, Dunne LE. Effects of the textile-sensor interface on stitched strain sensor performance. International Semantic Web Conference. 2019.

29. Weber M, Abigail CS, Tara B, et al. Development of a pressure measuring garment to understand how to quantify compression. 2020.

30. Vandycke S. MinStrain. Master's Thesis, Minneapolis, MN: University of Minnesota; 2018.

31. Logan LR, Hickman RR, Harris SR, et al. Single-subject research design: recommendations for levels of evidence and quality rating. Developmental Medicine \& Child Neurology. 2008;50(2):99-103.

32. StromBer. Klammerknoten für Angler. Own Work; 2008. 